\title{
HIGH POWER SEVENTH HARMONIC CONVERTER AT 20 GHZ*
}

\author{
M.A. LaPointe, ${ }^{1,2}$ V.P. Yakovlev, ${ }^{1}$ J.L. Hirshfield ${ }^{1,2}$ \\ ${ }^{1}$ Omega-P, Inc., 345 Whitney Ave, Suite 100, New Haven, CT 06511, USA \\ ${ }^{2}$ Yale University, Beam Physics Lab, P.O. Box 208124, New Haven, CT 06520-8124, USA
}

\section{Abstract}

A design is described of a $7^{\text {th }}$ harmonic converter utilizing a two-cavity system for producing RF power at $20 \mathrm{GHz}$. The converter uses a $250 \mathrm{kV}, 20 \mathrm{~A}$ electron beam injected into the first cavity tuned to the $\mathrm{TE}_{111}$ mode at $2.856 \mathrm{GHz}$. The beam is accelerated in this cavity via a cyclotron resonance interaction [1] using $8.5 \mathrm{MW}$ of RF power to an energy of $670 \mathrm{kV}$ with $99 \%$ efficiency. The accelerated beam passes to an output cavity tuned to the $\mathrm{TE}_{711}$ mode where simulations predict an output power of $4 \mathrm{MW}$ at $19.992 \mathrm{GHz}$.

\section{INTRODUCTION}

Harmonic converters can be a useful high-power if source technology for testing accelerator and microwave components at frequencies where a primary high power source does not yet exist. A two cavity $8^{\text {th }}$ harmonic converter was previously designed to generate power at $91 \mathrm{GHz}$.[2] The basic principle is to utilize an existing high power microwave tube (a SLAC XK-5 klystron for the converter described here) and up-convert the RF to a frequency of interest. For the device described here, $2.856 \mathrm{GHz}$ power is used to generate, via a seventh harmonic interaction, output power at $19.992 \mathrm{GHz}$.

\section{DESIGN}

The harmonic converter consists of four major subsystems: electron gun, rf system, magnetic system and beam collector. Fig. 1 depicts the arrangement.

\subsection{Electron Gun}

The electron gun is a $100 \mathrm{kV}, 10^{-6} \mathrm{~A}-\mathrm{V}^{-3 / 2}$, Pierce type diode gun with a $200 \mathrm{kV}$ post-acceleration stage. The ratio of the cathode voltage to the intermediate anode voltage can be varied by approximately $10 \%$ to allow flexibility in the voltage/current characteristics of the output beam (i.e. the beam current is not entirely determined by the terminal voltage). For this experiment the gun will be operated at $250 \mathrm{kV}$ with an output current of $20 \mathrm{~A}$. The calculated beam diameter at the entrance to the drive cavity is $2.5 \mathrm{~mm}$ with a moderate area compression ratio of 200:1.

\subsection{RF System}

The RF drive power for this experiment is supplied by a SLAC XK-5 klystron originally capable of $21 \mathrm{MW}$ at $2.856 \mathrm{GHz}$. The RF power required for this experiment is 8.5 MW, a value within the present capabilities of this klystron. The RF power is split by a $3 \mathrm{~dB}$ hybrid and sent to the two port, $90^{\circ}$ input coupler on the drive cavity where a rotating $\mathrm{TE}_{111}$ mode is launched. The cavity has a radius of $3.81 \mathrm{~cm}$ and a length of $7.37 \mathrm{~cm}$. Irises on the waveguide inputs, along with the beam input and output irises, are adjusted so as to give a loaded $\mathrm{Q}$ of approximately 130 .

The output cavity is operated in the $\mathrm{TE}_{711}$ mode at $19.992 \mathrm{GHz}$, the $7^{\text {th }}$ harmonic of the drive frequency. This cavity has a $2.06 \mathrm{~cm}$ radius and a length of $6.0 \mathrm{~cm}$. The predicted loaded Q is approximately 900. The generated $19.992 \mathrm{GHz} \mathrm{RF}$ power is then extracted axially through an output iris into an up-tapered section to decouple the beam from the RF and stop the interaction. The RF is then sampled by a wall probe to measure the frequency and power and finally dissipated on a RF absorber to prevent reflections back into the output cavity. An output coupler will be designed and installed once the initial proof-of-principle experiments are done.

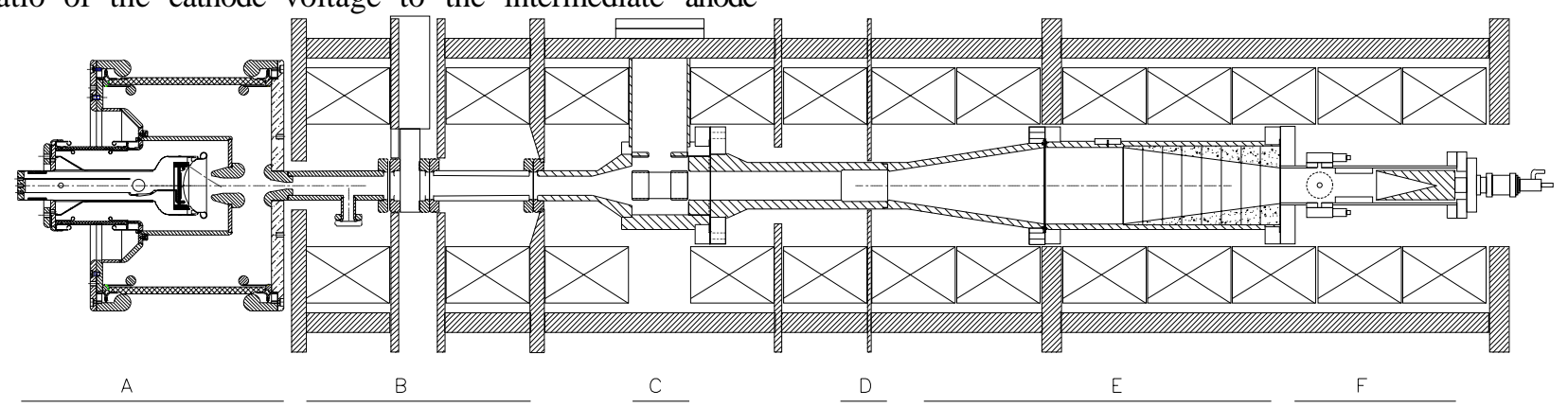

Figure 1: The harmonic converter showing the (A) electron gun, (B) lens doublet matching system, (C) the input coupler and $\mathrm{TE}_{111}$ cavity, (D) $\mathrm{TE}_{711}$ cavity, (E) extraction section and (F) beam dump. Also shown is the magnetic system of solenoid coils, flux cage and pole pieces.

*Research sponsored by the U.S. Department of Energy 


\subsection{Magnetic System}

The magnetic system consists of a series of solenoid coils and pole pieces to provide the magnetic field (see Fig. 2). After the beam exits the electron gun it first passes through a lens doublet which is used to match the electron beam into the first cavity. A two coil system is used to generate the 1450-2050 Gauss magnetic field with the proper gradient along the beam path so as to provide phase synchronism between the beam and the RF. Because of limitations due to the presence of the input couplers the magnetic field peaks after the cavity at 2610 Gauss. This results in the necessity of an additional pole piece between the two cavities as well as an extended beam tunnel in order to reduce the field to that required in the output cavity. The magnetic field requirements in the output cavity needed to match the synchronism conditions for the $\mathrm{TE}_{711}$ mode require a field decreasing from 2230-1840 Gauss. A mid-cavity pole piece is required to provide the necessary gradient.

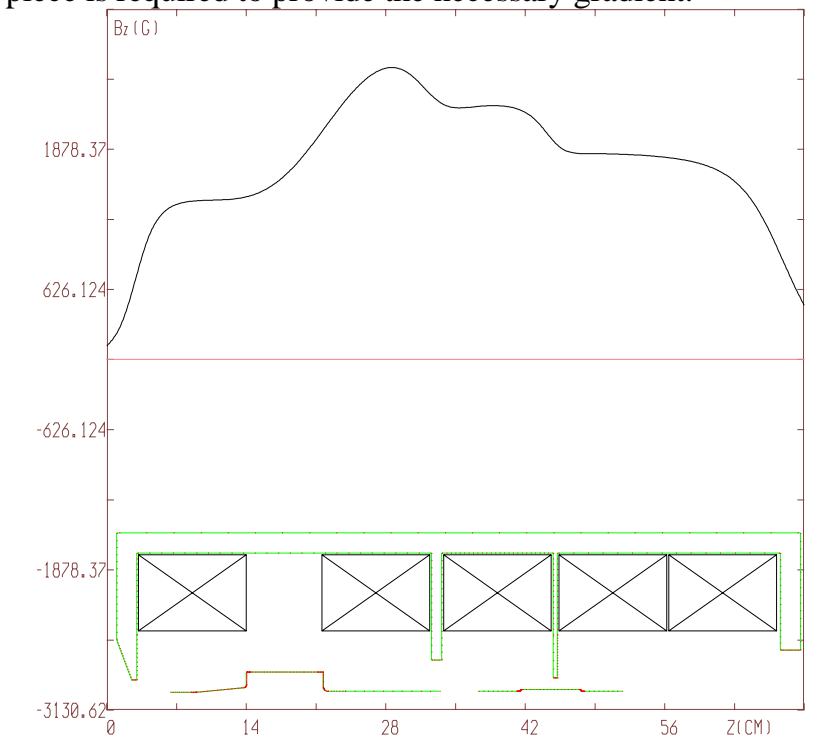

Figure 2: Magnetic system layout and magnetic field profile.

The balance of the magnetic system shown in Fig. 1 is for transport of the beam to the collector. Magnetic field strength in this region is such that no beam-RF interactions should take place.

\subsection{Beam Collector}

The beam collector is a copper faraday cup with a conical inside shape to provide for small angle incidence to reduce secondary emission, as well as to limit $\mathrm{x}$-ray production. The entrance diameter is $3.8 \mathrm{~cm}$ which is greater than the largest beam diameter anticipated in the device. The collector is electrically and thermally isolated from the main body of the tube by a ceramic insulated high current feed-through. This allows for measurement of the collected current and allows for calorimetry of the collected beam energy. Calibration is achieved by operating the tube without RF drive and measuring the un-accelerated beam. This also allows for a determination of beam interception during acceleration by noting any loss of current.

\section{SIMULATION RESULTS}

Simulations using the MGC code [3] were performed to optimize the parameters for the interaction cavities. The input beam, modeled using the SuperSAM code [4] with realistic parameters, had a diameter of $2.5 \mathrm{~mm}$ which is approximately $10 \%$ larger than the Brillouin limit. This $250 \mathrm{kV}, 20 \mathrm{~A}$ beam was used for the drive cavity simulation which had $8.5 \mathrm{MW}$ of $2.856 \mathrm{GHz}$ power in the $\mathrm{TE}_{111}$ mode. Simulations show that $99 \%$ of the $8.5 \mathrm{MW}$ drive power is absorbed by the electrons, resulting in a $670 \mathrm{kV}, 13.4 \mathrm{MW}$ beam (see Fig. 3). The maximum radius of the accelerated beam is well within the physical dimensions of apertures in the device so current interception is not anticipated.

The beam is then transported through the extended beam tunnel to the output cavity where it launches and interacts with the $\mathrm{TE}_{711}$ mode. The predicted $4 \mathrm{MW}$ of $19.992 \mathrm{GHz}$ power shown in the simulations represent a $47 \%$ RF conversion efficiency and a total conversion efficiency of $30 \%$.

As seen in Fig. 3 the energy spread is well behaved throughout the device with no increase until after the output cavity interaction. The simulation included transverse space charge effects.

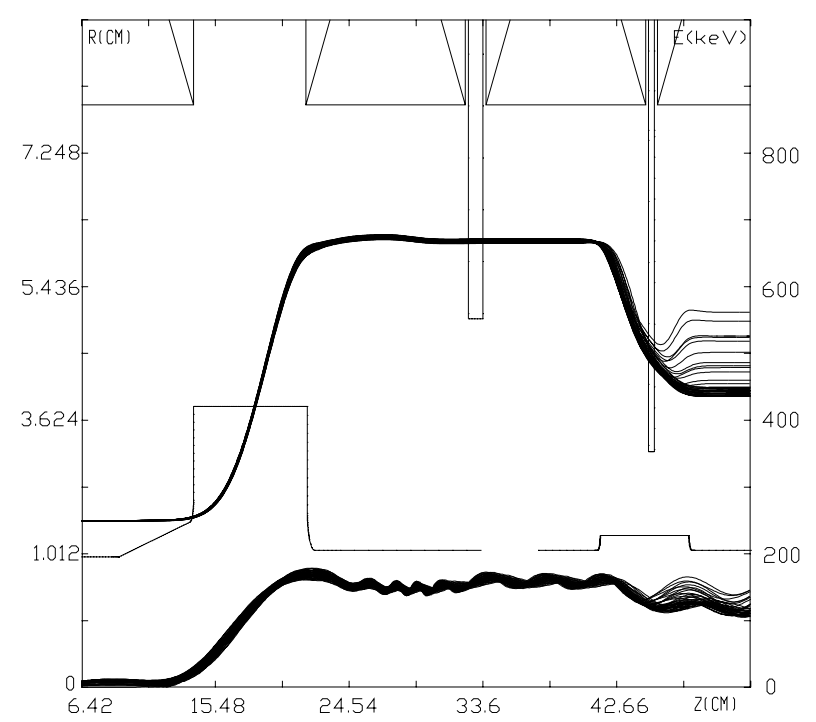

Figure 3: Simulation results from MGC code showing the development of the beam radius (bottom) and energy (top) in the device.

\section{STATUS}

The electron gun is currently operating and in use on another experiment. The input coupler and cavity sections have been fabricated and are currently undergoing cold testing before final machining and brazing. The absorber 
section, with the RF sampling point, has been fabricated and the Ceradyne RF absorbers are in hand. The beam dump/calorimeter is ready for installation. The solenoid coils for the magnet system are in hand, and the pole pieces and flux cage are being fabricated. The device is expected to be operating by end of Summer 2001.

\section{DISCUSSION}

The current limitation on the output power for the device presented here is the availability of $2.856 \mathrm{GHz} \mathrm{RF}$ power. Although the XK-5 klystron had a capability of over $21 \mathrm{MW}$ when first manufactured, the cathode is beginning to fail on the klystron in operation at the Beam Physics Lab. Currently only 12-15 MW of RF at full klystron beam power can be obtained. Thus the designed RF power for this experiment of $8.5 \mathrm{MW}$ was placed well within the currently available range of the XK-5 klystron.

If the full $20 \mathrm{MW}$ RF power of were available from the XK-5 klystron then one could contemplate what parameters might be achievable. First, if one assumes that only $95 \%$ of the $20 \mathrm{MW}$ is absorbed then the same initial beam is accelerated to $1.2 \mathrm{MeV}$ with a power of $24 \mathrm{MW}$. If we keep the same RF conversion efficiency of $47 \%$ then approximately $9.4 \mathrm{MW}$ would be generated at the $7^{\text {th }}$ harmonic. This would give a total conversion efficiency of $39 \%$. Realisation of this possibility would require simulations and study which have not yet been performed. Analysis for this case or even higher power levels (e.g. $65 \mathrm{MW}$ SLAC 5045 tube as RF source) is possible with the necessary redesign of the various subsystems.

The harmonic converter is not limited to either the frequency or harmonic number used for this experiment.
An $8^{\text {th }}$ harmonic converter [2] similar to the one described here was designed to produce $10 \mathrm{MW}$ of $91 \mathrm{GHz} \mathrm{RF}$ using the $11.4 \mathrm{GHz}$ magnicon at NRL [5]. The design of harmonic converters of this type, although not trivial, are straightforward and well within current state of the art in both design codes and manufacturing techniques. It is expected that such devices can find application in the evaluation of high-power accelerating structures and components at frequencies where a primary source does not yet exist.

\section{REFERENCES}

[1] M.A. LaPointe, R.B. Yoder, Changbiao Wang, A.K. Ganguly and J.L. Hirshfield, Phys. Rev. Lett. 76, 2718 (1996).

[2] J.L. Hirshfield, O.A. Nezhevenko, Changbiao Wang, V.P. Yakovlev, A.A. Bogdashov, V.L. Bratman, A.V. Chirkov, G.G. Denisov, A.N. Kuftin, S.V. Samsonov, and A.V. Savilov, "10 MW, W-Band RF Source for a High Gradient Linear Accelerator", EPAC2000, pp. 20722074. Vienna, 2000.

[3] V. Yakovlev, O. Danilov, O. Nezhevenko, V. Tarnetsky, Proceedings of 1995 Particle Accelerator Conference and International Conference on High Energy Accelerators, Dallas, 1995, pp.1569-1571.

[4] D.G. Myakishev, M.A. Tiunov and V.P. Yakovlev, "Code SuperSAM for Calculation of Electron Guns with High Beam Area Convergence", XV-th International Conference on High Energy Accelerators, 1992, Hamburg. Int. J. Mod. Phys. A (proc. Suppl.) 2B (1993), v-2, pp.915-917.

[5] O.A. Nezhevenko, et.al., "Status of X-Band Pulsed Magnicon Project," Present conference. 